\title{
Stochastic Economic Dispatch Using Bacterial Swarm Algorithm
}

\author{
M.S. Li, Y. Hu \\ School of Electric Power Engineering \\ South China University of Technology \\ Guangzhou, China
}

\author{
X. Zhang \\ The Chinese University of Hong Kong Shenzhen Research \\ Institute \\ Shenzhen, China
}

\begin{abstract}
This paper adopts a stochastic optimization method for solving the Security-Constrained Optimal Power Flow (SCOPF) problem with the consideration of distributed load variations in the grid. The objective function of the dispatch scheme aims to minimize fuel costs of the grid. Compared with conventional dispatch scheme, the computational complexity of proposed method is significantly increased. Therefore, this research adopts an improved Bacterial Swarm Algorithm (BSA) to solve the optimization. Compared with most Evolutionary Algorithms (EAs), BSA is more effective and has better convergence performance. The simulation studies reports the results obtained using an IEEE 30-bus system with uncertain load. A comparison between the results achieved using the proposed method and those obtained from conventional dispatch is given.
\end{abstract}

Keywords-economic dispatch; stochastic; bacterial swarm algorithm

\section{INTRODUCTION}

Economic dispatch has been intensively studied as a network constrained Optimal Power Flow (OPF) problem, since its introduction by Carpenter [1] in 1962. Generally, the economic dispatch problem aims to achieve the minimization of the fuel cost of a model of a power system, by adjusting the control variables, such as power and voltages of each generator, the tap ratios of transformers and the reactive power of volt-ampere reactive of the system, while satisfying a set of operational and physical constraints [2]. As a result, the economic dispatch problem is formulated as a non-linear constrained optimization problem.

Conventional dispatch studies assume that the model of the grid is invariant between the dispatch intervals, which are defined as deterministic dispatch [3]. However, the power system is significantly affected by uncertainty factors, including the renewable energy generators and distributed loads. Environmental uncertainties, such as weather conditions and climate, causes variation of loads. Therefore, it is necessary to develop an economic dispatch scheme for the gird, which is able to deal with the environmental uncertainties [4]. Conventional deterministic dispatch schemes usually consider deterministic objective functions and constraints. In such frames, the load is assumed to be invariant. The power consumed on each bus in deterministic dispatch is considered as a constant value, which is in conflict with actual system [5]. Thus, the control variables obtained is not reliable.
Most recent studies have focused on the uncertainties in the distributed load due to the effects of the unpredictable climate changing. To reduce the influence of such uncertainties, recently proposed stochastic dispatch frameworks minimize the generation cost of the scenario that is most likely to occur the future, and modify the constraints to accommodate other possible scenarios [6]. Some of the existing research has used commitment decisions and implemented multiple stages of preventive and corrective measures to address the uncertainties in the dispatch process. In this paper, we adopt a novel concept of stochastic dispatch, which considers the variations of distributed loads between dispatch actions. Different from deterministic dispatch, stochastic dispatch focuses on simultaneously optimizing the expectation and deviation of the fuel cost of the grid, which avoids the risk of an unpredictable operational status by introducing a mean-variance portfolio [7].

To solve the stochastic dispatch problem, a novel optimization algorithm, BSA, is introduced [8]. The BSA is inspired from the bacterial chemo taxis behavior described in Bacterial Foraging Algorithm (BFA). Moreover, BSA also describes further details of bacterial behaviors, and incorporates the mechanisms of quorum sensing. BSA models two bacterial behaviors: 1) Chemo taxis offer the basic search principle of BSA, which comprises of two basic foraging patterns, tumble and run. The biased random walk performing the local search. In the tumble process, the heading angle of each bacterium is described as a compound angle; 2) Quorum sensing enables BSA to escape from local optima. This is a two-fold operation that can either attract a bacterium to the optimal location or repel it away from the location where bacteria are concentrated. According to previous, BSA demonstrates a superior performance in comparison with other Evolutionary Algorithms (EAs).

\section{StOchastic ECONOMIC DisPatch}

The objective function of stochastic dispatch can be formulated as a minimization problem, described as follows:

$$
\begin{array}{r}
\min F(Y, X) \\
\text { s.t. } G(Y, X)=0 \\
H(Y, X)>0,
\end{array}
$$

Where $\mathrm{F}(\mathrm{Y}, \mathrm{X})$ is the objective function, which is concerned with fuel cost, $G(Y, X)$ is a set of equality constraints, and 
$\mathrm{H}(\mathrm{Y}, \mathrm{X})$ is a set of formulated inequality constraints. $\mathrm{Y}$ is the vector of dependent variables, which is expressed as:

$$
Y^{\top}=\left[P_{G_{1}} V_{L_{1}} \cdots V_{L N_{G}} Q_{G_{1}} \cdots Q_{G N_{G}} S_{1} \cdots S_{N_{E}}\right],
$$

Which includes the slack bus power $P_{G_{1}}$, the load bus voltage $\mathrm{V}_{\mathrm{L}}$, generator reactive power outputs $\mathrm{Q}_{\mathrm{G}}$, and the apparent power flow S.Xis the set of control variables:

$$
X^{\top}=\left[P_{G_{2}} \cdots P_{G N_{G}} V_{G_{1}} \cdots V_{G N_{G}} T_{1} \cdots T_{N_{T}} Q_{C_{1}} \cdots Q_{C N_{C}}\right]
$$

Which includes the generator real power output $\mathrm{P}_{G}$ except slack bus $\mathrm{P}_{\mathrm{G}_{1}}$; the generator voltages $\mathrm{V}_{\mathrm{G}}$, the transformer tap setting $T$, and the reactive power generations of var source $Q_{C}$. The detailed notations and formulation for equality constraints and inequality constraints are given in [4].

The objective, $\mathrm{F}$, is formulated to reducing the mathematical expectation and variance of the fuel cost to alleviate the uncertainty in the power system:

$$
F=\mathrm{E}\left[f_{\text {cost }}\right]+\lambda_{\text {Var }} \operatorname{Var}\left[f_{\text {cost }}\right]
$$

WhereE[ $[\cdot]$ is the mathematical expectation of the stochastic function; $\lambda_{V a r}$ is a weight to balance the mathematical expectation and variance; $\operatorname{Var}[\cdot]$ is the variance of the stochastic function; and $\mathrm{f}_{\text {cost }}$ is the fuel cost of the power system. The mathematical expectation of the fuel cost is expressed as:

$$
E\left[f_{\text {cost }}\right]=\frac{1}{N_{S}} \sum_{i=1}^{N_{S}} f_{\text {cost }_{i}}
$$

where $\mathrm{N}_{\mathrm{S}}$ is the number of scenario used in each objective function evaluation, $\mathrm{f}_{\text {cost }_{i}}$ is the fuel cost of the power system calculated with the $\mathrm{i}^{\text {th }}$ scenario. The variance of the fuel cost is expressed as:

$$
\operatorname{Var}\left[f_{\text {cost }}\right]=E\left[\left(f_{\text {cost }_{i}}-E\left[f_{\text {cost }}\right]\right)^{2}\right]
$$

The fuel cost of the $\mathrm{i}^{\text {th }}$ scenario is calculated as follow:

$$
\begin{gathered}
f_{\text {cost }_{i}}=\sum_{j=1}^{N_{G}} f_{\text {cost }_{i j}}, i=1,2, \cdots, N_{S} \\
f_{\text {cost }_{i j}}=a_{j}+b_{j} P_{G_{i j}}+c_{j} P_{G_{i j}}^{2}, j=1,2, \cdots, N_{G}
\end{gathered}
$$

In these equations, $\mathrm{N}_{\mathrm{S}}$ denotes the number of scenarios, $\mathrm{N}_{\mathrm{G}}$ denotes the number of generators, $f_{\text {cost }_{i j}}$ is the fuel cost $(\$ / h)$ of the $j^{\text {th }}$ generator at the ith scenario, $a_{j}, b_{j}$ and $c_{j}$ are fuel cost coefficients, and $\mathrm{P}_{\mathrm{G}_{i j}}$ is the real power output generated by the jth generator at the ith scenario.

The equality constraints $\mathrm{H}(\mathrm{Y}, \mathrm{X})$ are the power flow equations:

$$
\begin{array}{cr}
0=P_{G_{i}}-P_{D_{i}}-V_{i} \sum_{j \in N_{i}} V_{j}\left(G_{i j} \cos \theta_{i j}+B_{i j} \sin \theta_{i j}\right) & i \in \\
N_{0} & (11) \\
0=Q_{G_{i}}-Q_{D_{i}}-V_{i} \sum_{j \in N_{i}} V_{j}\left(G_{i j} \sin \theta_{i j}+B_{i j} \cos \theta_{i j}\right) & i \in \\
N_{\mathrm{PQ}}, & (12)
\end{array}
$$

The inequality constraints $\mathrm{G}(\mathrm{Y}, \mathrm{X})$ are the limits of the control variables and state variables, which can be formulated as:

$$
\mathrm{P}_{\mathrm{G}_{\mathrm{i}}}^{\min } \leq \mathrm{P}_{\mathrm{G}_{\mathrm{i}}}<\mathrm{P}_{\mathrm{G}_{\mathrm{i}}}^{\max }
$$

$$
\begin{gathered}
\mathrm{Q}_{\mathrm{G}_{\mathrm{i}}}^{\min } \leq \mathrm{Q}_{\mathrm{G}_{\mathrm{i}}}<\mathrm{P}_{\mathrm{G}_{\mathrm{i}}}^{\max } \\
\mathrm{Q}_{\mathrm{C}_{\mathrm{i}}}^{\min } \leq \mathrm{Q}_{\mathrm{C}_{\mathrm{i}}}<\mathrm{Q}_{\mathrm{C}_{\mathrm{i}}}^{\max } \\
\mathrm{T}_{\mathrm{k}}^{\min } \leq \mathrm{T}_{\mathrm{k}}<\mathrm{T}_{\mathrm{k}}^{\max }
\end{gathered}
$$

The load variations distributed across the system is described as Gaussian distribution. The real power consumed at the jth load bus is denoted by $\widehat{P}_{D_{j}}$, which is assumed to obey a Gaussian distribution with an expected value of $P_{D_{j}}$ and a standard deviation of $0.1 \mathrm{P}_{\mathrm{D}_{\mathrm{j}}}$. The probability density function of the distributed load variations is expressed as follows:

$$
P\left(\widehat{P}_{D_{j}}\right)=\frac{1}{\sqrt{2 \pi\left(0.1 P_{D_{j}}\right)^{2}}} \exp \left(-\frac{\left(\widehat{P}_{D_{j}}-P_{D_{j}}\right)^{2}}{2\left(0.1 P_{D_{j}}\right)^{2}}\right)
$$

\section{BACTERIAL SWARM OPTIMIZER}

Suppose the pth bacterium, in the tumble-run process of thekth iteration, has a current position $\mathrm{X}_{\mathrm{p}}^{\mathrm{k}}$. The objective of the optimization is to find the minimum of $F\left(X_{p}^{k}\right)$. The bacterium also has a rotation angle $\varphi_{\mathrm{p}}^{\mathrm{k}}=\left(\varphi_{\mathrm{p} 1}^{\mathrm{k}}, \varphi_{\mathrm{p} 2}^{\mathrm{k}}, \cdots \varphi_{\mathrm{p}(\mathrm{n}-1)}^{\mathrm{k}}\right)$ and a tumble length $\mathrm{D}_{\mathrm{p}}^{\mathrm{k}}\left(\varphi_{\mathrm{p}}^{\mathrm{k}}\right)=\left(\mathrm{d}_{\mathrm{p} 1}^{\mathrm{k}}, \mathrm{d}_{\mathrm{p} 2}^{\mathrm{k}}, \cdots \mathrm{d}_{\mathrm{pn}}^{\mathrm{k}}\right)$, which can be calculated from $\varphi_{\mathrm{p}}^{\mathrm{k}}$ via a polar-to-cartesian coordinate transform:

$$
\begin{gathered}
d_{\mathrm{p} 1}^{\mathrm{k}}=\prod_{\mathrm{i}=1}^{\mathrm{n}-1} \cos \left(\varphi_{\mathrm{pi}}^{\mathrm{k}}\right), \\
\mathrm{d}_{\mathrm{pj}}^{\mathrm{k}}=\sin \left(\varphi_{\mathrm{p}(\mathrm{j}-1)}^{\mathrm{k}}\right) \prod_{\mathrm{i}=\mathrm{p}}^{\mathrm{n}-1} \cos \left(\varphi_{\mathrm{pi}}^{\mathrm{k}}\right) \mathrm{j}=2,3, \cdots, \mathrm{n}-1, \\
\mathrm{~d}_{\mathrm{pn}}^{\mathrm{k}}=\sin \left(\varphi_{\mathrm{p}(\mathrm{n}-1)}^{\mathrm{k}}\right)
\end{gathered}
$$

In the tumble-run process of the $\mathrm{k}^{\text {th }}$ iteration, the $\mathrm{p}^{\text {th }}$ bacterium generates a random rotation angle, which falls in the range of $\left[0, \varphi_{\max }\right]$. A tumble action takes place in an angle expressed as:

$$
\widehat{\varphi}_{\mathrm{p}}^{\mathrm{k}}=\varphi_{\mathrm{p}}^{\mathrm{k}}+\frac{\mathrm{r}_{1} \varphi_{\max }}{2}
$$

Where $r_{1}$ is a uniform random sequence with a range of $[-1,1]$. The run action immediately follows the tumble action. Because the run action will be performed more than once, the position $\mathrm{X}_{\mathrm{p}}^{\mathrm{k}}$ is recorded as $\mathrm{X}_{\mathrm{p}}^{\mathrm{k}, 0}$, which indicates the position of the pth bacterium at the beginning of the kt iteration.

Once the angle is determined by the tumble step, the bacterium will run for a maximum of $\mathrm{N}_{\mathrm{c}}$ run steps. If at the $\mathrm{N}_{\mathrm{f}}^{\text {th }}$ run step, the bacterium finds a position which has a better fitness value than the current one, the run process also stops. The position of the pthbacterium is updated at the hthrun step in the following way:

$$
\widehat{\mathrm{X}}_{\mathrm{p}}^{\mathrm{k}, \mathrm{h}}=\widehat{\mathrm{X}}_{\mathrm{p}}^{\mathrm{k}, \mathrm{h}-1}+\mathrm{r}_{2} \mathrm{D}_{\mathrm{p}}^{\mathrm{k}}\left(\widehat{\varphi}_{\mathrm{p}}^{\mathrm{k}}\right)
$$

Wherer $_{2}$ is a normally distributed random number, and $\widehat{X}_{\mathrm{p}}^{\mathrm{k}, \mathrm{h}}$ is the position of the $\mathrm{p}^{\text {th }}$ bacterium after the hth run step. For convenience of description, the position of the pthbacterium beginning immediately after the tumble-run process of the kth iteration is denoted by $\widehat{\mathrm{X}}_{\mathrm{p}}^{\mathrm{k}, \mathrm{N}_{\mathrm{f}}}$. 
Inspired by PSO, the positions of the bacteria moving by attraction are updated as follows:

$$
X_{p}^{k}=\widehat{X}_{p}^{k, N_{f}}+r_{3}\left(X_{\text {best }}-\widehat{X}_{p}^{k, N_{f}}\right)
$$

where $_{3}$ is a normally distributed random number with a range of $[-1,1]$, which describes the strength of bacterial attraction, and $\mathrm{X}_{\text {best }}$ indicates the position of the current best global solution updated after the evaluation of each function.

In BSA, a small number of the bacteria are randomly selected to be repelled. To measure the degree of repelling, a repelling rate is defined by $\zeta$, lemph\{i.e.\}, in each iteration, $100 \zeta$ percent of the bacteria are processed by repelling. Accordingly the attraction rate is $100(1-\zeta)$ percent. The repelling process is based on therandom searching principle. If the $\mathrm{p}^{\text {th }}$ bacterium shifts into the repelling process, a random angle is generated. The bacterium is thereby moved to a random position following this angle in the search space, which can be described as:

$$
\mathrm{X}_{\mathrm{p}}^{\mathrm{k}}=\widehat{\mathrm{X}}_{\mathrm{p}}^{\mathrm{k}, \mathrm{N}_{\mathrm{f}}}+\mathrm{r}_{4} \mathrm{D}_{\mathrm{p}}^{\mathrm{k}}\left(\widehat{\varphi}_{\mathrm{p}}^{\mathrm{k}}+\pi / 2\right)
$$

Wherer $_{4}$ is a normally distributed random sequence.

\section{Simulation STUdies}

The simulation studies are undertaken on the well-studied IEEE 30-bus system. This model represents a portion of the American Electric Power System. The model comprises 30 buses, 6 generators, and 40 branches. The fuel cost coefficients of the generators, given in (10), are listed in Table I. The BSA is evaluated and compared with Genetic Algorithm (GA) [9] and Particle Swarm Optimizer (PSO) [10]. For the PSO parameters, the inertia weight is 0.73 , and the acceleration factors are both 2.05 , as recommended in [10]. The maximum number of function evaluations for all algorithms is set to $3 \times 10^{6}$. The maximum number of scenario taken in the optimization process, $N_{S}$, is set to 200 .

TABLE I. FUEL COST COEFFICIENTS IN THE IEEE 30-BUS SYSTEM.

\begin{tabular}{|c|c|c|c|}
\hline Generator & $a$ & $b$ & $c$ \\
\hline 1 & 0 & 2 & 0.02 \\
\hline 2 & 0 & 1.75 & 0.0175 \\
\hline 3 & 0 & 1 & 0.0625 \\
\hline 4 & 0 & 3.25 & 0.0083 \\
\hline 5 & 0 & 3 & 0.025 \\
\hline 6 & 0 & 3 & 0.025 \\
\hline
\end{tabular}

Table II lists the mean and variance of the fuel costs obtained by BSA, GA and PSO. The experimental results demonstrated that proposed BSA (807.2853) outperforms GA 809.6017 and PSO 808.6747 on the objective of mean fuel cost. Meanwhile, the fuel cost optimized by BSA also has the smallest standard deviation (10.5451). Thus, the grid operational policy obtained by BSA is robust.
TABLE II.MEAN AND VARIANCE OF THE FUEL COSTS OBTAINED BY STOCHASTIC DISPATCH.

\begin{tabular}{|c||c|c|c|}
\hline Algorithm & $\begin{array}{l}\text { Mean fuel cost } \\
(\$ / \mathrm{h})\end{array}$ & $\begin{array}{l}\text { Standard deviation } \\
\text { of fuel cost }(\$ / \mathrm{h})\end{array}$ & $\begin{array}{l}\text { Computational } \\
\text { time (seconds) }\end{array}$ \\
\hline \hline BSA & $\mathbf{8 0 7 . 2 8 5 3}$ & $\mathbf{1 0 . 5 4 5 1}$ & 571 \\
GA & 809.6017 & 10.9191 & 640 \\
PSO & 808.6747 & 11.6947 & $\mathbf{5 4 4}$ \\
\hline
\end{tabular}

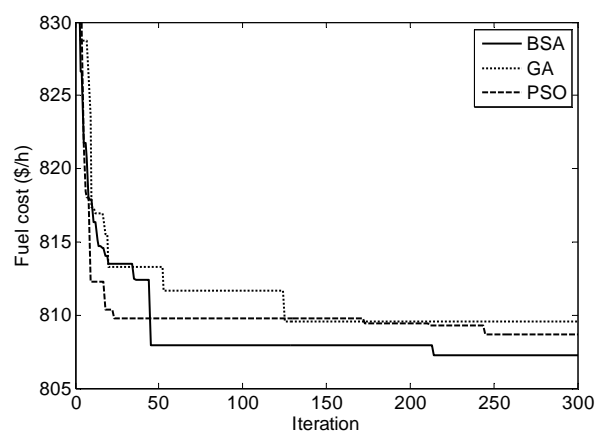

FIGURE I. CONVERGENCE PROGRESS.

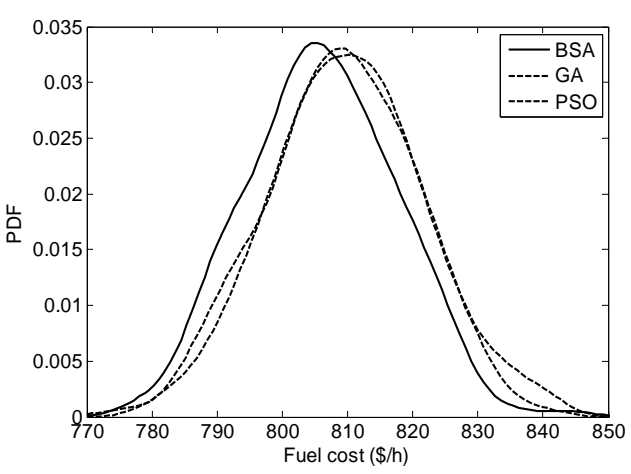

FIGURE II. PDF OF FUEL COST.

The convergence progresses of BSA, GA and PSO are illustrated in Figure 1. It can be found that although BSA converges slowly in the early period of the searching, the quorum sensing prevents the premature result in the optimization, and leads to a better performance in the late stage. Figure 2 shows the distribution of the fuel cost estimated by these three algorithms. The fuel cost estimated by BSA has a small mean value and standard deviation.

\section{CONCLUSION}

This paper has adopted a novel stochastic model for economic dispatch in an environment that considers distributed load uncertainties. Simulation studies have been conducted on an IEEE 30-bus system with the simultaneous objectives of minimizing the mean value and the standard deviation of fuel cost. The simulation results indicate that BSA provides a more reliable solution set for power system dispatch than GA and PSO due to its excellent convergence performance.

\section{ACKNOWLEDGEMENTS}

This research is jointly supported by National Natural Science Foundation of China (51307062) and Guangdong Innovative Research Team Program (No. 201001N0104744201). 


\section{REFERENCES}

[1] Carpentier, J., Contribution to the economic dispatch problem, Bull. Soc.Franc. Elect, 1962, pp. 431-447.

[2] Yu Xia; Ghiocel, S.G.; Dotta, D.; Shawhan, D.; Kindle, A.; Chow, J.H., "A Simultaneous Perturbation Approach for Solving Economic Dispatch Problems With Emission, Storage, and Network Constraints," Smart Grid, IEEE Transactions on , vol.4, no.4, pp.2356,2363, Dec. 2013

[3] Chamba, M.S.; Ano, O., "Economic Dispatch of Energy and Reserve in Competitive Markets Using Meta-heuristic Algorithms," Latin America Transactions, IEEE (Revista IEEE America Latina), vol.11, no.1, pp.473,478, Feb. 2013

[4] Li, M.S.; Ji, T.Y.; Wu, Q.H.; Xue, Y.S., "Stochastic Optimal Power Flow using a Paired-Bacteria Optimizer," Power and Energy Society General Meeting, 2010 IEEE, vol., no., pp.1,7, 25-29 July 2010

[5] Chang, C.S.; Fu, W., "Stochastic multiobjective generation dispatch of combined heat and power systems," Generation, Transmission and Distribution, IEE Proceedings- , vol.145, no.5, pp.583,591, Sep 1998

[6] M.S. Li, Q.H. Wu, T.Y. Ji, H. Rao, Stochastic multi-objective optimization for economic-emission dispatch with uncertain wind power and distributed loads, Electric Power Systems Research, Volume 116, November 2014, Pages 367-373

[7] Xiangyu Cui; Xun Li; Duan Li, "Unified Framework of Mean-Field Formulations for Optimal Multi-Period Mean-Variance Portfolio Selection," Automatic Control, IEEE Transactions on , vol.59, no.7, pp.1833,1844, July 2014

[8] M.S. Li, T.Y. Ji, W.J. Tang, Q.H. Wu, J.R. Saunders, Bacterial foraging algorithm with varying population, Biosystems, Volume 100, Issue 3 , June 2010, Pages 185-197

[9] Leung, H.C.; Chung, T.S., "Optimal power flow with a versatile FACTS controller by genetic algorithm approach," Advances in Power System Control, Operation and Management, 2000. APSCOM-00. 2000 International Conference on , vol.1, no., pp.178,183 vol.1, 30 Oct.-1 Nov. 2000

[10] Clerc, M.; Kennedy, J., "The particle swarm - explosion, stability, and convergence in a multidimensional complex space," Evolutionary Computation, IEEE Transactions on, vol.6, no.1, pp.58,73, Feb 2002 